\title{
Observance des cédules de remplacement des lentilles jetables et des silicone hydrogels par les patients et les praticiens au Canada
}

\author{
PAR DORIS RICHTER, MASc, OD; KATHY DUMBLETON, MSc, MCOptom, FAA0; SARAH GUTHRIE, PhD; \\ CRAIG WOODS, PhD, MCOptom, FAA0; LYNDON JONES, PhD, FCOptom, FAAO; DESMOND FONN, MOptom, FAAO
}

\section{Introduction}

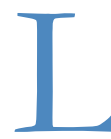

a plupart des lentilles souples prescrites aujourd'hui doivent être remplacées à intervalles réguliers qui vont d'un jour à un mois. Une enquête mondiale réalisée sur les habitudes de prescription des lentilles cornéennes en $2008^{1}$ révèle que les nouveaux porteurs de lentilles souples au Canada ont le plus fréquemment $(75 \%)$ reçu des lentilles de remplacement d'un mois, suivies des lentilles de remplacement de deux semaines $(14 \%)$ et des lentilles journalières jetables $(11 \%)$. Des études ont indiqué que le remplacement fréquent des lentilles cornéennes à base d'hydrogel diminue l'incidence des dépôts et des complications et accroît la satisfaction générale des patients, comparativement aux lentilles qui sont portées

\section{RÉSUMÉ}

Objet : Évaluer la fréquence de remplacement (FR) des silicone hydrogels (SH) et des lentilles cornéennes journalières jetables (JJ) au Canada recommandée actuellement par les optométristes, déterminer les taux de non-conformité aux recommandations par les optométristes et les patients, et analyser les raisons de la non-conformité.

Méthodes : Des trousses de questionnaires ont été envoyées aux optométristes au Canada qui avaient accepté de participer à l'enquête. Les patients ont répondu à des questions d'ordre démographique et à d'autres sur leurs habitudes de port de lentilles cornéennes, notamment la FR recommandée et effectivement suivie. Les optométristes devaient fournir des renseignements sur les lentilles et la FR qu'ils recommandaient. Cinquante-huit optométristes ont renvoyé 654 questionnaires, dont 578 ont pu être analysés.

Résultats : Soixante-dix pour cent des patients étaient des femmes présentant un âge médian de 32 ans. La répartition par type de lentille s'établissait comme suit : 18 \% JJ, 35 \% SH de deux semaines et 47 \% SH d'un mois. Six pour cent des lentilles étaient à port prolongé. Les patients ont indiqué porter leurs lentilles pendant une période médiane de 12 heures par jour; dans le cas des JJ, la médiane était de cinq jours/semaine, tandis qu'elle a été de sept jours/semaine pour les SH (deux semaines et un mois). La FR recommandée par les optométristes a été différente de celle des fabricants dans $6 \%$ des
JJ, $35 \%$ des SH de deux semaines et $2 \%$ des SH d'un mois. Parmi les patients qui n'ont suivi ni les recommandations du fabricant ni celles de l'optométriste, $12 \%$ portaient des JJ, $43 \%$, des SH de deux semaines et $31 \%$, des SH d'un mois. Les patients qui ne respectaient pas les recommandations avaient pour la plupart oublié quel jour ils devaient remplacer leurs lentilles. Cinquante-six pour cent des patients ont précisé qu'un système de rappel les aiderait à se conformer à la FR. Un pourcentage plus élevé de patients ont respecté la FR parce quiils avaient confiance dans leur optométriste.

Conclusions : En général, les optométristes ont adopté la fréquence de remplacement recommandée par les fabricants dans le cas des lentilles JJ et des SH d'un mois, mais ils ont souvent recommandé des intervalles plus longs pour les SH de deux semaines. (e sont les porteurs de lentilles IJ qui ont le plus suivi les recommandations, mais ce sont les porteurs de SH de deux semaines qui se sont le moins conformés aux recommandations. La conformité s'accroitrait sans doute s'il y avait un système de rappel ou si l'optométriste insistait sur les risques susceptibles de découler de la non-observation des règles.

Mots clés : Silicone hydrogel, lentilles journalières jetables, lentilles cornéennes, fréquence de remplacement, conformité 
plus longtemps. ${ }^{2-8}$ De tels avantages reposent en partie sur la conformité à la fréquence de remplacement recommandée. Des études antérieures avaient indiqué que les patients ne respectaient pas toujours les calendriers de remplacement, ${ }^{9-12}$ mais aucune de ces études n'a porté précisément sur les lentilles en silicone hydrogel qui représentent actuellement près de $50 \%$ des nouvelles lentilles souples prescrites au Canada. ${ }^{1,13}$

Cette étude a pour but d'évaluer la fréquence de remplacement (FR) recommandée par les optométristes au Canada pour ce qui est des silicone hydrogels $(\mathrm{SH})$ et des lentilles cornéennes journalières jetables (JJ). Nous avons aussi déterminé les taux de non-conformité des optométristes et des patients à la fréquence de remplacement recommandée par les fabricants (FRRF) et examiné les raisons d'une telle non-conformité.

\section{Méthodes}

\section{Plan de l'étude}

L'étude a suivi le plan utilisé dans une étude similaire qui avait porté sur les praticiens oculovisuels aux États-Unis et sur leurs porteurs de lentilles $\mathrm{JJ}$ et $\mathrm{SH} .{ }^{14}$ Une invitation à participer à cette étude volontaire a été envoyée par courrier électronique à près de 2500 optométristes au Canada. L'Association canadienne des optométristes a invité ses membres à participer à l'étude.

Le Centre de recherche en lentille cornéenne (CRLC) a envoyé par la poste des trousses de questionnaires aux optométristes participants. La lettre d'explication que les optométristes ont reçue les enjoignait de demander à leurs 20 prochains patients portant des lentilles cornéennes souples (soit des JJ, soit des SH de deux semaines ou d'un mois) de répondre au questionnaire. Les patients qui acceptaient de participer à l'enquête pouvaient le faire en tout anonymat. Après avoir rempli le questionnaire, le patient le cachetait dans une enveloppe sur laquelle l'optométriste inscrivait le type de lentille, la puissance de chaque œil et la fréquence de remplacement qu'il recommandait. L'optométriste renvoyait ensuite tous les questionnaires remplis (dans leurs enveloppes scellées) au CRLC dans une enveloppe port payé. Pour préserver l'anonymat des optométristes au CRLC, les optométristes n'étaient pas identifiés sur les questionnaires retournés.

L'autorisation sur le plan éthique a été obtenue par l'entremise du Bureau de l'éthique de la recherche de l'Université de Waterloo et l'étude s'est conformée aux principes de la Déclaration de Helsinki.

\section{L'enquête}

Le questionnaire de trois pages était composé d'une série de questions sur le patient et sur ses antécédents concernant le port de lentilles cornéennes, notamment sur la fréquence de remplacement des lentilles qui lui avait été recommandée et celle qu'il a effectivement suivie. Des questions ont aussi été posées pour déterminer pourquoi les patients n'observaient pas la fréquence de remplacement recommandée et pour examiner les directives de l'optométriste au sujet de la fréquence de remplacement des lentilles.

\section{Analyse des données}

Le cas échéant, les analyses de données ont été faites à l'aide de Statistica 8.0 (StatSoft Inc. Tulsa, $\mathrm{OK})$. Les données sont présentées sous forme de pourcentages ou de moyennes \pm l'écart-type. Lorsque des estimations d'intervalles sont en jeu, elles représentent des limites de confiance à $95 \%$ de l'estimation.

\section{Résultats}

\section{Répondants}

Cinquante-huit optométristes (52\% des optométristes qui avaient exprimé leur intérêt de participer à l'enquête) ont renvoyé 654 questionnaires de patient (29\% de tous les questionnaires envoyés). Les données de 578 patients répondants ont pu être analysées (88\% des questionnaires remplis reçus). Les autres questionnaires ne contenaient pas suffisamment d'information pour permettre de les analyser ou ont été remplis par des patients qui portaient des lentilles d'une marque non incluse dans l'enquête.

\section{Marques et puissances des lentilles}

Le tableau 1 présente les marques des lentilles portées par les patients et consignées par les optométristes. 
Dix-huit pour cent des lentilles portées étaient des lentilles journalières jetables (JJ), $35 \%$ étaient des silicone hydrogels de remplacement de deux semaines ( $\mathrm{SH}$ de deux semaines) et $47 \%$ étaient des silicone hydrogels de remplacement d'un mois (SH d'un mois).

Soixante-six pour cent des patients participants ont indiqué la même marque de lentilles que leur optométriste. Dans $5 \%$ des cas, le type de lentille ne correspondait pas, tandis que $29 \%$ des patients n'étaient pas certains de la marque de lentilles qu'ils portaient.

\section{Données démographiques et habitude de port des lentilles}

Soixante-dix pour cent des questionnaires analysés ont été remplis par des femmes. L'âge moyen de tous les patients s'établit à $33,7 \pm 12,7$ ans (la fourchette va de 14 à 77 ans) et leur âge médian, à 32 ans. Les patients participants avaient déjà porté des lentilles cornéennes; le nombre moyen d'années de port d'un type ou l'autre de lentilles cornéennes est de 12,1 \pm 9,4 (la fourchette va de 0,1 à 49 ans), avec une durée médiane de 10 ans.

Les patients ont indiqué porter leurs lentilles $5,6 \pm 1,9$ jours par semaine (fourchette d'un à sept jours), avec une médiane de cinq jours pour les lentilles $\mathrm{JJ}$ et de sept jours pour les lentilles SH de deux semaines et d'un mois. Le pourcentage de patients portant des lentilles moins de sept jours par semaine a été plus élevé chez les porteurs de lentilles JJ (70\%,

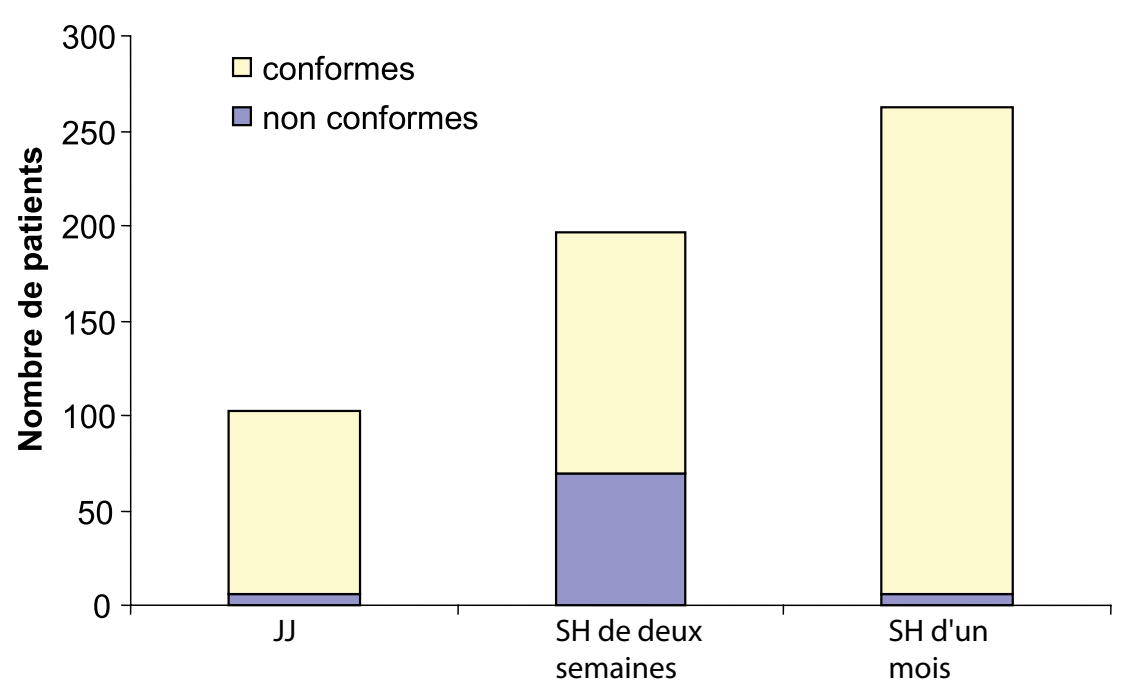

Figure 1: Conformité de l'optométriste à la fréquence de remplacement recommandée par le fabricant

\section{TABLEAU 1}

\section{RÉPARTITION DES MARQUES DE LENTILLES PORTÉES}

\begin{tabular}{|c|c|c|c|}
\hline & Nombre & Type de lentilles (\%) & $\%$ dans le groupe \\
\hline \multicolumn{4}{|l|}{ Lentilles d'un jour } \\
\hline 1 Day Acuvue (et Moist) & 27 & 26 & \multirow{10}{*}{18} \\
\hline 1 Day Acuvue for Astigmatism & 1 & 1 & \\
\hline Dailies (et Aqua, Aqua Plus) & 56 & 54 & \\
\hline Dailies Toric & 8 & 8 & \\
\hline Dailies Progressive & 3 & 3 & \\
\hline Proclear 1 Day & 3 & 3 & \\
\hline Freshlook 1-Day & 2 & 2 & \\
\hline Biomedics 1 Day Toric & 2 & 2 & \\
\hline Biomedics 1 Day & 1 & 1 & \\
\hline Soflens 1 Day & 1 & 1 & \\
\hline \multicolumn{4}{|l|}{ Lentilles de deux semaines } \\
\hline Acuvue Advance & 49 & 24 & \multirow{4}{*}{35} \\
\hline Acuvue Advance for Astigmatism & 38 & 19 & \\
\hline Acuvue Oasys & 100 & 49 & \\
\hline Acuvue Oasys for Astigmatism & 16 & 8 & \\
\hline \multicolumn{4}{|l|}{ Lentilles d'un mois } \\
\hline Air Optix / Air Optix Aqua & 91 & 34 & \multirow{7}{*}{47} \\
\hline Air Optix Astigmatism & 14 & 5 & \\
\hline Night \& Day & 54 & 20 & \\
\hline Biofinity & 25 & 9 & \\
\hline PureVision & 28 & 10 & \\
\hline PureVision Toric & 34 & 13 & \\
\hline PureVision Multifocal & 25 & 9 & \\
\hline
\end{tabular}


TABLEAU 2

FRÉQUENCE DE REMPLACEMENT RECOMMANDÉE PAR L'OPTOMÉTRISTE

\begin{tabular}{|c|c|c|c|c|c|c|c|c|c|}
\hline & \multicolumn{9}{|c|}{ FRRO } \\
\hline Groupe de lentilles & $1 \mathrm{~J}$ & $2 J$ & $3-6 J$ & 15 & $2 S$ & 35 & $1 \mathrm{M}$ & $2 \mathrm{M}$ & $\geq 3 \mathrm{M}$ \\
\hline 1 jour $(n=102)$ & $94.1 \%$ & 0 & 0 & 0 & $1.0 \%$ & 0 & $4.9 \%$ & 0 & 0 \\
\hline 2 semaines $(n=197)$ & 0 & 0 & 0 & 0 & $65.0 \%$ & $1.5 \%$ & $32.0 \%$ & $1.5 \%$ & 0 \\
\hline 1 mois $(n=262)$ & $0.4 \%$ & 0 & 0 & $0.4 \%$ & $6.5 \%$ & 0 & $90.5 \%$ & $0.4 \%$ & $1.9 \%$ \\
\hline
\end{tabular}

\section{TABLEAU 3}

\section{FRÉQUENCE DE REMPLACEMENT RECOMMANDÉE PAR L'OPTOMÉTRISTE (DÉCLARÉE PAR LE PATIENT)}

\begin{tabular}{|l|c|c|c|c|c|c|c|c|c|c|}
\hline & \multicolumn{10}{c|}{ FRRP } \\
\hline Groupe de lentilles & 1 J & 2J & 3-6J & 15 & 25 & 35 & 1 M & $2 M$ & $\geq 3$ M & AR \\
\hline 1 jour $(n=103)$ & $81.6 \%$ & $1.0 \%$ & $1.0 \%$ & 0 & $1.0 \%$ & 0 & $2.9 \%$ & 0 & 0 & $12.6 \%$ \\
\hline 2 semaines $(n=202)$ & $1.5 \%$ & 0 & $0.5 \%$ & $2.5 \%$ & $53.5 \%$ & $4.0 \%$ & $34.2 \%$ & $1.5 \%$ & $0.5 \%$ & $2.0 \%$ \\
\hline 1 mois $(n=269)$ & $1.1 \%$ & 0 & 0 & $1.5 \%$ & $7.1 \%$ & $1.9 \%$ & $79.6 \%$ & $4.1 \%$ & $1.9 \%$ & $3.0 \%$ \\
\hline
\end{tabular}

TABLEAU 4

\section{FRÉQUENCE DE REMPLACEMENT PAR RAPPORT À LA FRÉQUENCE DE REMPLACEMENT RECOMMANDÉE}

\begin{tabular}{|l|c|c|c|c|}
\hline \multicolumn{4}{|c|}{ Remplacement des lentilles cornéennes par rapport à ce qui a été recommandé } \\
\hline Groupe de lentilles & Toujours & Souvent & Parfois & Rarement \\
\hline 1 jour & $76 \%$ & $13 \%$ & $4 \%$ & $7 \%$ \\
\hline 2 semaines & $29 \%$ & $38 \%$ & $17 \%$ & $15 \%$ \\
\hline 1 mois & $36 \%$ & $32 \%$ & $19 \%$ & $13 \%$ \\
\hline Toutes lentilles & $41 \%$ & $31 \%$ & $16 \%$ & $13 \%$ \\
\hline
\end{tabular}

$60 \%-79 \%$ que de lentilles de deux semaines $(39 \%, 32 \%-48 \%)$ ou d'un mois $(39 \%, 33 \%-45 \%)$. La durée moyenne de port des lentilles à port quotidien s'est établie à $11,9 \pm 3,4$ heures par jour (la fourchette va de 2 à 19 heures), avec une médiane de 12 heures. Dans l'ensemble, $6 \%$ des patients ont indiqué porter leurs lentilles 24 heures par jour; ce pourcentage a varié selon le groupe de lentilles, les taux allant de $3 \%$ (1 \% à $9 \%)$ pour les lentilles JJ, à $<1 \%(0 \%-3 \%)$ pour les lentilles de deux semaines et à $11 \%(7 \%-15 \%)$ pour les lentilles d'un mois.
Conformité des optométristes à la fréquence de remplacement recommandée

\section{Données obtenues des} optométristes

Le tableau 2 présente la répartition des réponses en ce qui concerne la fréquence de remplacement recommandée par l'optométriste (FRRO) pour chaque groupe de lentilles. La fréquence de remplacement a été jugée conforme aux recommandations du fabricant (FRRF) si l'intervalle entre les remplacements était inférieur ou égal à la fréquence recommandée. Les cases en surbrillance dans le tableau 2 représentent les FRRO qui étaient conformes.
Un énorme pourcentage de FRRO a été plus long que les FRRF dans le cas des lentilles de deux semaines $(35 \%, 28 \%-42 \%)$. Les taux de non-conformité ont été moins élevés pour les lentilles JJ $(6 \%, 2 \%-13 \%)$ et les lentilles d'un mois $(2 \%, 1 \%-5 \%)$. Ces résultats sont résumés à la figure 1 . Des FRRO plus courtes ont été déclarées pour $7 \%$ des porteurs de lentilles d'un mois (5 \% $11 \%$ ). Dans la plupart de ces cas, il s'agissait de lentilles AIR OPTIX (également connues sous le nom de $\mathrm{O}_{2}$ OPTIX, Ciba Vision).

Le taux de non-conformité des FRRO a été plus élevé dans le cas 
des lentilles pour astigmates $(24 \%$, $17 \%-33 \%$ que des lentilles sphériques $(11 \%, 8 \%-15 \%)$.

\section{Données obtenues des patients}

Le tableau 3 présente la répartition des FR recommandées par l'optométriste que les patients ont déclarée (FRRP). Les cases en surbrillance représentent les FRRP qui sont conformes aux FRRF pour le type de lentille en question. Les lettres «AR » signifient qu'aucune recommandation n'a été faite.

La plupart des porteurs de lentilles de deux semaines et d'une semaine avaient reçu de leur optométriste une FR recommandée, mais $13 \%(7,2 \%-21,0 \%)$ des porteurs de lentilles $\mathrm{JJ}$ ont indiqué ne pas avoir eu une telle recommandation. Dans $78 \%$ des cas, la FRRP correspondait à la FRRO. Quatorze pour cent ne correspondaient pas et $8 \%$ avaient une ou deux FR manquantes ou aucune recommandation.

\section{Conformité des patients à la fréquence de remplacement recommandée}

Trois questions étaient destinées à évaluer dans quelle mesure les patients ont effectivement suivi la fréquence de remplacement recommandée. Le tableau 4 présente la répartition des réponses à la première question qui cherchait à savoir à quelle fréquence les patients remplaçaient leurs lentilles cornéennes par rapport à ce qui leur avait été recommandé. Un pourcentage beaucoup plus élevé de porteurs de lentilles JJ que de porteurs de lentilles de deux

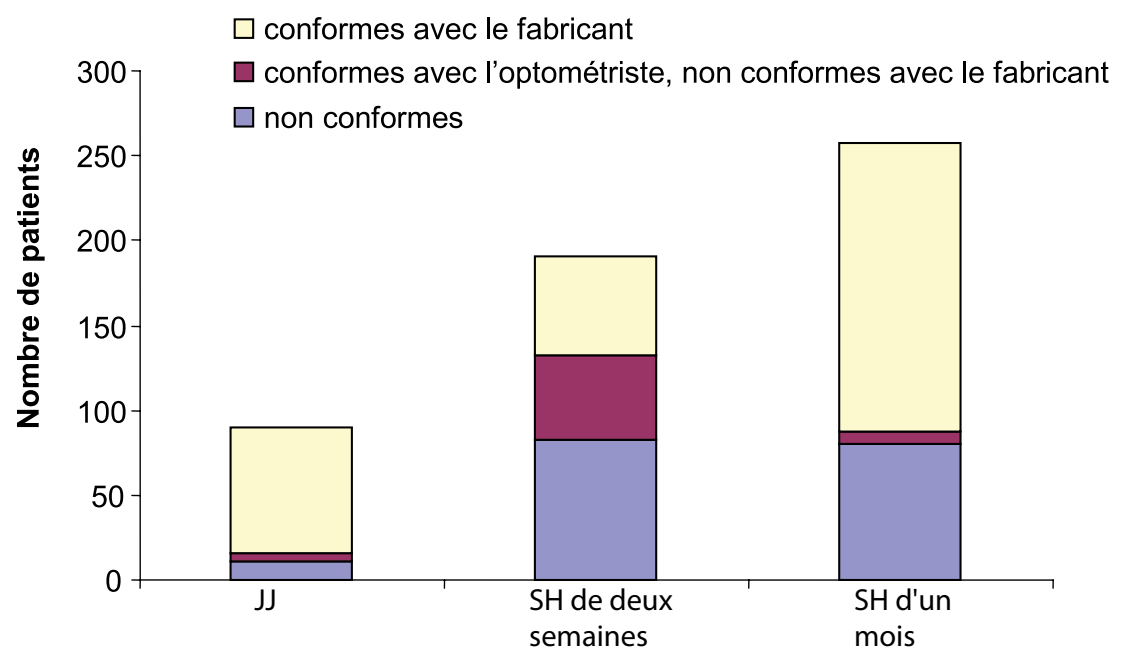

Figure 2: Conformité du patient à la FR recommandée par l'optométriste et le fabricant.

semaines et d'un mois ont indiqué « toujours » remplacer leurs lentilles à la fréquence recommandée.

À la deuxième question, les patients devaient indiquer la raison principale pour laquelle ils portaient leurs lentilles au-delà de la durée recommandée. Dans l'ensemble, $35 \%(30,9 \%-39,1 \%)$ des patients ayant répondu à la question ont indiqué qu'ils ne portaient jamais leurs lentilles plus longtemps que l'intervalle recommandé. Cette conclusion a été différente selon le groupe, puisque $73 \%(62,0 \%-$ $81,1 \%$ ) des porteurs de lentilles JJ, $22 \%(16,9 \%-28,9 \%)$ des porteurs de lentilles de deux semaines et $31 \%(25,5 \%-37,2 \%)$ des porteurs de lentilles d'un mois ont déclaré ne jamais dépasser la FR recommandée. La raison la plus courante invoquée par les porteurs de lentilles JJ pour dépasser la durée recommandée était " pour économiser de l'argent ». Pour les porteurs de lentilles de deux semaines et d'un mois, la raison la plus fréquente était « avoir oublié quel jour ils devaient les remplacer».

Enfin, on a demandé aux patients après combien de jours ou de mois ils remplaçaient leurs lentilles. Une fréquence de remplacement (FR) supérieure à une journée était jugée non conforme dans le cas de lentilles JJ; une FR supérieure à 17 jours était jugée non conforme dans le cas de lentilles de deux semaines, et une fréquence de plus de 31 jours était considérée comme non conforme pour ce qui est de lentilles d'un mois. Dans l'ensemble, $44 \%$ des répondants portaient leurs lentilles plus longtemps que la FRRF. Ce pourcentage a varié selon le groupe de lentilles, les taux de non-conformité s'établissant à $18 \%(10,8 \%$ $27,6 \%)$ dans le cas des lentilles JJ, à $69 \%(62,0 \%-75,5 \%)$ pour les lentilles de deux semaines et à $34 \%$ $(28,5 \%-40,4 \%)$ pour les lentilles d'un mois. 
Étant donné que les optométristes ne respectent pas toujours la FRRF, surtout dans le cas des lentilles de remplacement de deux semaines, quelques patients peuvent sembler ne pas se conformer alors que, en réalité, ils ne font que suivre la recommandation de leur optométriste. Il serait par conséquent plus approprié de considérer les patients comme non conformes uniquement s'ils ne respectent ni la FRRF ni la FRRP. Vingt-six pour cent des patients qui ne suivaient pas la FRRF ont cependant observé la recommandation qu'ils se rappelaient avoir eue de leur optométriste. Lorsqu'on a inclus ces patients dans le groupe en conformité, le taux global de non-conformité des patients s'est fixé à $32 \%(28,4 \%-$ $36,5 \%)$; ̀̀ $12 \%(6,6 \%-21,2 \%)$ dans le cas des porteurs de lentilles JJ; à $43 \%(36,4 \%-50,8 \%)$ chez les porteurs de lentilles de deux semaines; et à $31 \%(25,6 \%-37,2 \%)$ pour ce qui est des porteurs de lentilles d'un mois. La figure 2 résume les données sur la conformité des patients.

\section{Systèmes de rappel}

Dans l'ensemble, $56 \%(50,4 \%$ $58,7 \%$ des patients étaient d'avis qu'un système de rappel les aiderait à suivre la fréquence de remplacement recommandée. Ce pourcentage a été moins élevé chez les porteurs de JJ (35\%, 26,2 \% $45,7 \%$ que chez les porteurs de lentilles de deux semaines (61 \%, $53,6 \%-67,4 \%$ ) ou chez les porteurs de lentilles d'un mois $(57 \%, 50,8 \%$ $63,1 \%)$. Les patients pouvaient choisir à partir d'une liste de systèmes de rappel ceux qu'ils jugeaient utiles. Sept pour cent des patients ont choisi

\section{TABLEAU 5}

\section{ACCORD SUR LES ÉNONCÉS SELON LE GROUPE DE CONFORMITÉ}

\begin{tabular}{|c|c|c|c|}
\hline \multicolumn{4}{|c|}{ Réponse: D'accord ou fortement d'accord } \\
\hline Énoncé & Conforme & Non conforme & $\begin{array}{l}\text { Écart entre conforme } \\
\text { et non conforme } \\
\text { (IC de } 95 \%)\end{array}$ \\
\hline $\begin{array}{l}\text { Si mon professionnel de la vue me dit de prendre soin de } \\
\text { mes lentilles cornéennes, je le fais }\end{array}$ & $94 \%$ & $77 \%$ & $\begin{array}{c}17 \% \\
(10,8 \% \text { to } 24,9 \%)\end{array}$ \\
\hline $\begin{array}{l}\text { Mon professionnel de la vue m'a clairement expliqué le } \\
\text { calendrier de remplacement de mes lentilles. }\end{array}$ & $93 \%$ & $84 \%$ & $\begin{array}{c}9 \% \\
(3,2 \% \text { to } 16,1 \%)\end{array}$ \\
\hline $\begin{array}{l}\text { Je suis le calendrier de remplacement recommandé parce } \\
\text { que j'ai une confiance totale dans mon professionnel de } \\
\text { la vue. }\end{array}$ & $87 \%$ & $51 \%$ & $\begin{array}{c}36 \% \\
(27,7 \% \text { to } 44,7 \%)\end{array}$ \\
\hline $\begin{array}{l}\text { Je n'ose jamais déroger à mon calendrier de remplacement } \\
\text { de mes lentilles. }\end{array}$ & $57 \%$ & $16 \%$ & $\begin{array}{c}41 \% \\
(32,8 \% \text { to } 48,4 \%)\end{array}$ \\
\hline $\begin{array}{l}\text { Je suis le calendrier de remplacement recommandé } \\
\text { pour éviter les complications avec mes yeux. }\end{array}$ & $78 \%$ & $38 \%$ & $\begin{array}{c}41 \% \\
(31,7 \% \text { to } 49,0 \%)\end{array}$ \\
\hline $\begin{array}{l}\text { Je suis plus susceptible de suivre le calendrier de } \\
\text { remplacement recommandé si mes lentilles sont } \\
\text { inconfortables. }\end{array}$ & $81 \%$ & $79 \%$ & $\begin{array}{c}2 \% \\
(-5,1 \% \text { to } 10,2 \%)\end{array}$ \\
\hline $\begin{array}{l}\text { La qualité de ma vision m'indique qu'il est temps } \\
\text { de remplacer mes lentilles. }\end{array}$ & $56 \%$ & $72 \%$ & $\begin{array}{c}-15 \% \\
(-23,6 \% \text { to }-6,2 \%)\end{array}$ \\
\hline $\begin{array}{l}\text { Si je ne remplace pas mes lentilles selon la fréquence } \\
\text { recommandée, j'ai des problèmes de vision. }\end{array}$ & $42 \%$ & $31 \%$ & $\begin{array}{c}11 \% \\
\text { (2,4\% to } 19,6 \%)\end{array}$ \\
\hline $\begin{array}{l}\text { Le risque d'une infection oculaire grave m'amènerait plus } \\
\text { certainement à suivre le calendrier de remplacement } \\
\text { recommandé. }\end{array}$ & $87 \%$ & $83 \%$ & $\begin{array}{c}4 \% \\
(-2,7 \% \text { to } 11,1 \%)\end{array}$ \\
\hline $\begin{array}{l}\text { J'utilise un calendrier ou un autre système pour me faire } \\
\text { penser de remplacer mes lentilles selon la fréquence } \\
\text { recommandée par mon professionnel de la vue. }\end{array}$ & $45 \%$ & $21 \%$ & $\begin{array}{c}25 \% \\
(15,9 \% \text { to } 32,3 \%)\end{array}$ \\
\hline
\end{tabular}


un calendrier sur papier, $20 \%$, un message par cellulaire ou un message texte, $19 \%$, un rappel par courrier électronique, et $29 \%$, l'affichage d'un compte à rebours sur l'étui de leurs lentilles. Enfin, $24 \%$ ont indiqué qu'ils trouveraient utile de fixer un jour particulier chaque semaine ou chaque mois.

\section{Résultats selon le groupe de conformité des patients}

Nous avons analysé plus en profondeur les données relatives aux patients conformes et non conformes afin de découvrir s'il y avait des associations possibles entre les données et l'état de non-conformité ou les raisons pouvant l'expliquer.

\section{Données démographiques et habitudes de port des lentilles} L'âge moyen des patients non conformes était de 32,7 (30,9 - 34,5) \pm 11,9 ans, ce qui est similaire à l'âge moyen des patients conformes : $34,1(32,7-35,4) \pm 13,1$ ans. Trente pour cent des femmes et $37 \%$ des hommes n'ont pas respecté les recommandations. Le nombre total moyen d'années de port de lentilles était très élevé autant chez les patients conformes que non conformes (11,7 et 13,0 ans respectivement). Un pourcentage plus élevé de patients portant des lentilles pour corriger un astigmatisme n'ont pas suivi les recommandations (40\%, $31,4 \%-50,0 \%$ ) comparativement aux patients portant des lentilles sphériques $(30 \%, 26,2 \%-35,2 \%)$.

\section{Opinions des patients}

Un pourcentage plus élevé de patients conformes $(87 \%)$ que de patients non conformes (58\%) ont jugé qu'il était « extrêmement important » ou 《important» de remplacer leurs lentilles dans les délais recommandés.

L'enquête a posé une série de questions sur les interactions entre le patient et son optométriste. La majorité des patients conformes et non conformes estimaient qu'il était « important » ou « très important » que leur optométriste leur explique les risques associés à une nonconformité aux règles $(76 \%)$, mais un pourcentage plus élevé de patients conformes (70 \%) que de patients non conformes $(55 \%)$ jugeaient important que l'optométriste leur explique en détail le calendrier de remplacement.

On a ensuite demandé aux patients s'ils étaient d'accord ou non sur dix énoncés portant sur les professionnels des soins oculovisuels et sur le port de lentilles cornéennes. Le tableau 5 présente les écarts dans les pourcentages de patients conformes et non conformes ayant répondu être « d'accord » ou «fortement d'accord » avec les énoncés. Pour bon nombre des énoncés, un pourcentage plus élevé de patients conformes ont répondu être « d'accord » ou «fortement d'accord».

\section{Pratiques de nettoyage des lentilles}

Trente-quatre pour cent des patients portant des lentilles de deux semaines ou d'un mois ne connaissaient pas le nom du système de nettoyage qu'ils utilisaient. Les pourcentages sont similaires chez les patients considérés conformes (35\%) et non conformes (32\%). Un pourcentage élevé de patients considérés conformes et non conformes jugeaient « important » ou « extrêmement important » de nettoyer leurs lentilles tous les jours ( $90 \%$ et $86 \%$ respectivement). La plupart des patients ont indiqué qu'ils remplaçaient leur étui à lentilles au moins tous les six mois. Les pourcentages de patients considérés conformes et non conformes déclarant remplacer leur étui seulement une fois l'an ou « jamais » se sont établis respectivement à $18 \%$ et $22 \%$.

\section{Discussion}

Le but principal de cette étude était de savoir si les optométristes et les patients se conformaient à la fréquence de remplacement recommandée pour les lentilles cornéennes JJ de même que pour les lentilles cornéennes $\mathrm{SH}$ de deux semaines et d'un mois au Canada. L'enquête s'est déroulée de manière que l'optométriste ne puisse connaitre les réponses de ses patients et aussi de façon que les coordonnateurs de l'étude ne puissent connaître l'identité des optométristes. On espérait qu'un tel anonymat suscite des réponses honnêtes aux questions de l'enquête.

La cohorte de l'étude se composait de patients canadiens et les résultats indiquent qu'ils sont représentatifs de la population de porteurs de lentilles cornéennes au Canada pour ce qui est de leur répartition par sexe et par âge. ${ }^{1}$ 
Bien que des optométristes de neuf provinces eussent accepté de participer, nous ne connaissons pas la répartition des questionnaires retournés par province. Il est possible que les résultats de l'enquête ne reflètent pas les caractéristiques régionales des cabinets d'optométrie au Canada.

Quarante-sept pour cent des répondants ont porté des lentilles SH d'un mois, $35 \%$, des lentilles SH de deux semaines et $18 \%$, des lentilles JJ. Le pourcentage de patients portant des lentilles moins de sept jours par semaine était le plus élevé dans le groupe de lentilles JJ. Il est clair que les lentilles JJ sont pratiques pour les patients qui ne désirent pas porter leurs lentilles tous les jours.

Les résultats de l'étude révèlent que les optométristes recommandent des intervalles de remplacement plus longs que la FRRF à environ $35 \%$ de leurs patients portant des $\mathrm{SH}$ de remplacement de deux semaines, mais qu'ils suivent les recommandations pour la plupart de leurs patients portant des SH d'un mois et des JJ. Environ $13 \%$ des porteurs de lentilles JJ ont indiqué n'avoir reçu aucune recommandation de leur optométriste, mais cela ne signifie pas nécessairement qu'on ne leur a pas dit de jeter les lentilles après les avoir portées une journée. Il est simplement possible que ces instructions n'aient pas été interprétées comme des recommandations d'une fréquence de remplacement, surtout si les lentilles n'étaient pas portées tous les jours. Dans $14 \%$ des cas, la FR recommandée indiquée par le patient ne correspondait pas à la FR recommandée déclarée par l'optométriste. Diverses raisons peuvent expliquer ces disparités. En effet, les optométristes ont $\mathrm{pu}$ se sentir obligés de consigner la bonne FRRF sur l'enveloppe, même si l'enquête se déroulait dans l'anonymat, plutôt que la FR qu'ils avaient effectivement recommandée à leurs patients. Les patients ne se sont peut-être pas rappelé exactement les recommandations que leur avait données leur optométriste. Il est également possible qu'une partie de ces patients portaient en réalité un type de lentille différent de celui que l'optométriste avait consigné; en effet, dans $5 \%$ des cas, la marque de lentille déclarée par l'optométriste a été différente de celle que le patient avait déclarée. De plus, $29 \%$ des patients n'étaient pas certains de la marque de leurs lentilles.

Bon nombre de patients ont effectivement déclaré une FR qui ne respectait pas celle du fabricant ni celle qu'ils se rappelaient avoir reçue de leur optométriste. Le taux de non-conformité a été le moins élevé chez les porteurs de lentilles JJ $(12 \%)$. Les porteurs de lentilles de deux semaines ont présenté le taux de non-conformité le plus élevé (43\%), suivis des porteurs de lentilles d'un mois (31\%). Des résultats similaires, assujettis à la marge d'erreur de la présente étude, ont été obtenus dans une enquête antérieure qui avait examiné la conformité à la fréquence de remplacement de lentilles classiques aux États-Unis et au Canada. ${ }^{11}$

Lorsqu'on combine le taux de nonconformité des optométristes et des patients, on constate qu'environ
$44 \%$ des porteurs de lentilles JJ ou de lentilles $\mathrm{SH}$ de deux semaines ou d'un mois ne remplacent pas leurs lentilles selon les recommandations du fabricant.

Le pourcentage relativement peu élevé de patients portant leurs lentilles JJ plus qu'une journée n'est peut-être pas sans conséquence lorsqu'on sait que des cas de kératite à Acanthamocba ont été signalés chez des patients qui réutilisaient des lentilles journalières jetables. ${ }^{15,16}$ En revanche, on ne connaît pas les conséquences de porter une lentille $\mathrm{SH}$ plus longtemps que la FRRF puisqu'aucune étude n'a encore examiné cette question. Une étude réalisée à Singapour a révélé que l'utilisation de lentilles au-delà de leur date de remplacement recommandée accroîtrait la probabilité de contracter une kératite à Fusarium ${ }^{17}$; cependant, cette étude portait sur de nombreux types de lentilles, si bien que le risque est susceptible de varier selon le matériau de la lentille.

Soixante-dix-huit pour cent des patients ont déclaré qu'il était " extrêmement important » ou 《important » de remplacer leurs lentilles à la date prévue. Il n'est sans doute pas surprenant que les patients considérés non conformes soient moins susceptibles que les patients conformes de croire qu'il était important de remplacer les lentilles dans les délais prévus (58\% contre $87 \%$ ).

Plus de la moitié des patients portant des lentilles $\mathrm{SH}$ de deux semaines et d'un mois ont indiqué qu'il serait utile de recourir à un système de rappel pour le remplacement des lentilles. Il est intéressant de signaler que $45 \%$ des patients 
qui respectaient la fréquence de remplacement recommandée utilisaient un calendrier ou un autre système de rappel, comparativement à seulement $21 \%$ des patients considérés non conformes.

L'interaction entre un patient et son optométriste peut être importante pour établir la conformité du fait que la majorité des patients jugeaient « important » ou « très important » que leur optométriste leur explique les risques associés à une non-conformité à la FR. Quelques écarts entre les patients considérés conformes et non conformes sont apparus à partir des réponses données à quelques-uns des énoncés inclus dans le sondage. Un pourcentage beaucoup plus élevé de patients dits conformes ont indiqué suivre la fréquence de remplacement recommandée parce qu'ils avaient une confiance totale dans leur professionnel de la vue $(87 \%$ contre $51 \%)$ et parce que cela entraînait moins de problèmes oculovisuels $(78 \%$ contre $38 \%$ ). Cela semblerait vouloir dire que les patients se conforment à la FR parce qu'ils ne veulent pas subir les risques perçus d'une non-conformité et parce qu'ils font confiance à l'information qu'ils ont reçue de leur optométriste à ce sujet. Par contre, un pourcentage plus élevé de patients non conformes ont été d'accord sur l'énoncé selon lequel « la qualité de ma vision m'indique qu'il est temps de remplacer mes lentilles cornéennes » (72 \% contre $56 \%$ ). Quelques patients peuvent ne pas respecter la fréquence recommandée parce qu'ils accordent moins d'importance aux risques potentiels d'une non- conformité, choisissant plutôt de remplacer leurs lentilles d'après les symptômes qu'ils éprouvent.

D'autres études traitant de la conformité aux règles concernant l'usure et le soin des lentilles cornéennes ont constaté des différences entre les patients considérés comme conformes et non conformes; cependant elles ont surtout examiné la conformité aux règles de nettoyage plutôt qu'à la fréquence de remplacement. ${ }^{18,19}$ La conformité est une question complexe et les tentatives de cerner des prédicteurs de conformité n'ont en général pas donné beaucoup de succès. ${ }^{20}$

Aucune preuve convaincante dans notre étude ne nous indique que l'âge ou le sexe pourrait être un signe de conformité à la FR des lentilles. Les patients qui avaient besoin de lentilles pour corriger un problème d'astigmatisme ont eu moins tendance à suivre les recommandations de remplacement que les patients qui portaient une lentille sphérique. Le coût plus élevé des lentilles cornéennes toriques pourrait inciter des patients à prolonger l'intervalle de remplacement de leurs lentilles pour économiser de l'argent. Nous avons examiné les pratiques de nettoyage des lentilles chez les porteurs de lentilles $\mathrm{SH}$ de deux semaines et d'un mois. Il ne semble pas y avoir une corrélation forte entre les patients qui ne respectent pas la FR et ceux qui tendent à négliger les procédures de nettoyage des lentilles. Il est raisonnable de supposer que les patients jugent qu'une non-conformité aux procédures d'hygiène des lentilles comporte un risque plus élevé que le fait de ne pas respecter la fréquence de remplacement des lentilles. Nous aurions besoin de données supplémentaires sur les pratiques réelles de nettoyage des lentilles des patients pour vérifier cette hypothèse.

\section{Conclusion}

Notre étude a examiné le comportement des patients et des optométristes en ce qui concerne la FR des lentilles cornéennes de prescription courante au Canada. En général, les optométristes ont recommandé des FR conformes aux recommandations des fabricants pour ce qui est des lentilles JJ et $\mathrm{SH}$ d'un mois, mais ils ont souvent recommandé des intervalles plus longs dans le cas des lentilles $\mathrm{SH}$ de deux semaines. Les patients qui ont le plus suivi les règles portaient des lentilles JJ et ceux qui ont le moins respecté la FR portaient des lentilles SH de deux semaines. Plus de la moitié des porteurs de lentilles $\mathrm{SH}$ de deux semaines ou d'un mois qui ne respectaient pas la fréquence de remplacement de leurs lentilles ont expliqué leur comportement par le fait qu'ils ne se rappelaient plus le jour où ils devaient remplacer leurs lentilles. Un système de rappel chez ces patients pourrait les aider à se conformer à la FR. Plus de la moitié des porteurs de lentilles $\mathrm{JJ}$ qui ne remplaçaient pas leurs lentilles à la date prévue le faisaient pour économiser de l'argent. Il serait sans doute utile dans ces cas que l'optométriste expose à ses patients les risques de ne pas se conformer à la fréquence de remplacement. 


\section{Remerciements}

\section{Ciba Vision Canada a contribué}

financièrement à cette étude. Nous désirons remercier la Dre Etty Bitton, professeure agrégée et directrice des stages externes, École d'optométrie - Université de Montréal et l'Association canadienne des optométristes pour leur aide avec cette étude.

\section{Correspondance:}

Dre Doris Richter

Centre de recherche en lentille cornéenne École d'optométrie

Université de Waterloo

Waterloo ON

N2L 3 G1 Canada

Tél. : $519888-4742$

Télec. : 519 884-8769

dbrichte@uwaterloo.ca

\section{Références}

1. Morgan PB, Woods C, Tranoudis I, et al. International contact lens prescribing in 2008, Contact Lens Spectrum 2009; 24;2: 28-32.

2. Poggio EC, Abelson MB. Complications and symptoms with disposable daily wear contact lenses and conventional soft daily wear contact lenses, CLAO J 1993; 19;2: 95-102.

3. A study of the complications induced by conventional and disposable contact lenses, CLAO J 1994; (2): 103-108.

4. Pritchard N, Fonn D, Weed K. Ocular and subjective responses to frequent replacement of daily wear soft contact lenses, CLAO J 1996; 22;1: 53-59.

5. Solomon OD, Freeman MI, Boshnick EL, et al., A 3-year prospective study of the clinical performance of daily disposable contact lenses compared with frequent replacement and conventional daily wear contact lenses, CLAO J 1996; 22;4: 250-257.

6. Porazinski AD, Donshik PC. Giant papillary conjunctivitis in frequent replacement contact lens wearers: a retrospective study, CLAO J 1999; 25 (3): 142-147.

7. Suchecki JK, Ehlers WH, Donshik PC, et al. A comparison of contact lensrelated complications in various daily wear modalities, CLAO J 2000; 26;4: 204-213.

8. Brennan NA, Coles M-LC. Deposits and symptomatology with soft contact lens wear, Int Contact Lens Clin 2000; 27 (3): 75-100.

9. Smith SK. Patient noncompliance with wearing and replacement schedules of disposable contact lenses, J Am Optom Assoc 1996; 67;3: 160-164.

10. Coopersmith L, Weinstock FJ. Current recommendations and practice regarding soft lens replacement and disinfection, CLAO J 1997; 23;3: 172-176.

11. Jones L, Dumbleton K, Fonn D, et al. Comfort and compliance with frequent replacement soft contact lenses, Optom Vis Sci 2002; 79;12s: 259.

12. Morgan P. Contact lens compliance and reducing the risk of keratitis, Optician 2007; 234;6109: 20-25.

13. Morgan PB, Woods C, et al. International contact lens prescribing in 2007, Contact Lens Spectrum 2008; 23;1: 36-41.

14. Dumbleton K, Woods C, Jones L, et al. Patient and practitioner compliance with silicone hydrogel and daily disposable lens replacement in the United States of America, Eye \& CL; 35;4: 164-171.

15. Woodruff S.A., et Dart J.K.G. Acanthamoeba keratitis occurring with daily disposable contact lens wear, $\mathrm{Br} \mathrm{J}$ Ophthalmol 1999; 83: 1088-1089.

16. Niyadurupola N, Illingworth CD. Acanthamoeba keratitis associated with misuse of daily disposable contact lenses, Cont Lens Anterior Eye 2006; 269-271.

17. Saw SM, Ooi PL, Tan DT, et al. Risk factors for contact lens-related fusarium keratitis: a case-control study in Singapore, Arch Ophthalmol 2007; 125;5: 611-617.

18. Radford C, Woodward EG, et Stapleton F. Contact lens hygiene compliance in a university population, J BCLA, 1993; 16(3) 105-111.

19. Claydon BE, Efron N, Woods C. A prospective study of non-compliance in contact lens wear, J. Br. Contact Lens Assoc 1996; 19:133-140.

20. Efron N. The truth about compliance, Contact Lens And Anterior Eye 1997; 20;3: $79-86$.

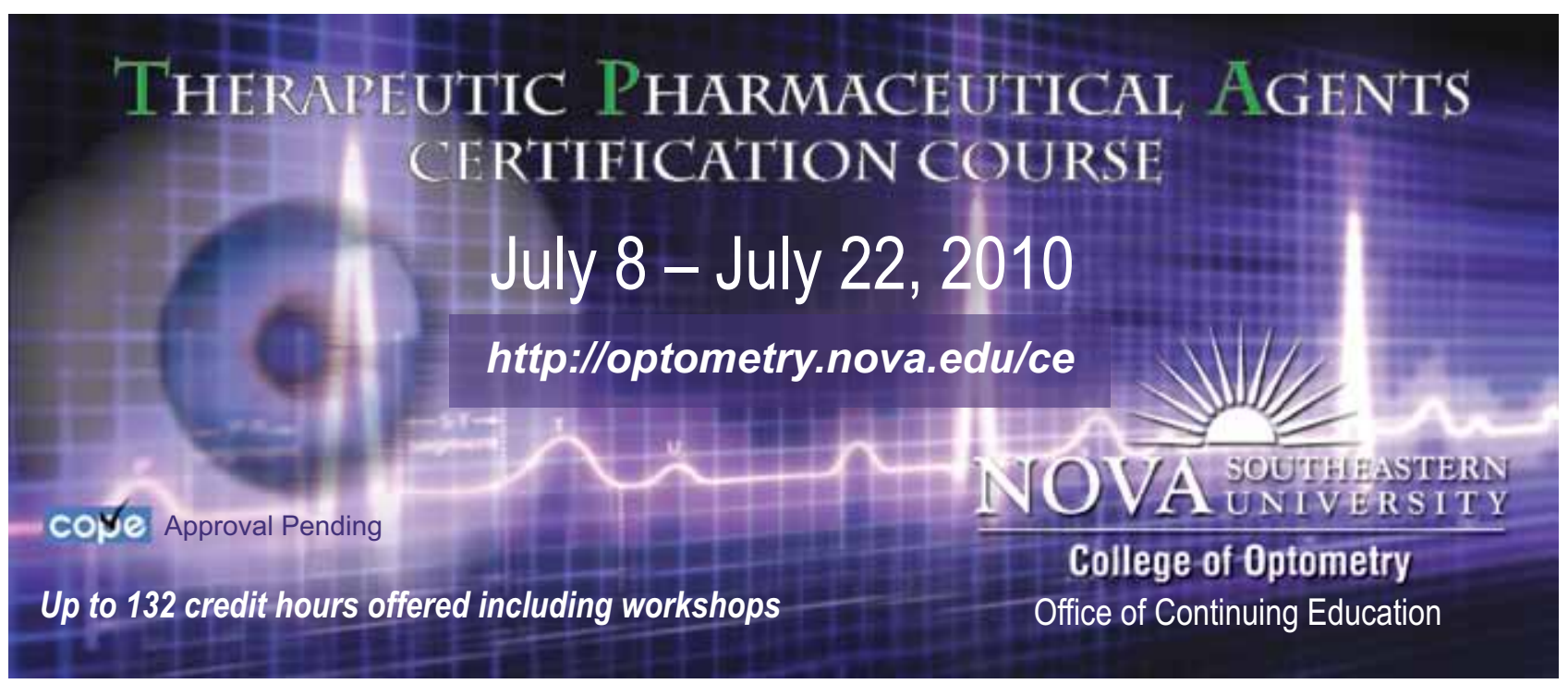

